\title{
CTX-M-15 extended-spectrum $\beta$-lactamases in Enterobacteriaceae in the intensive care unit of Tlemcen Hospital, Algeria
}

Z. Baba Ahmed, ${ }^{1}$ A. Ayad, ${ }^{1}$ E. Mesli, ${ }^{1}$ Y. Messai, ${ }^{2}$ R. Bakour ${ }^{2}$ and M. Drissi ${ }^{1}$

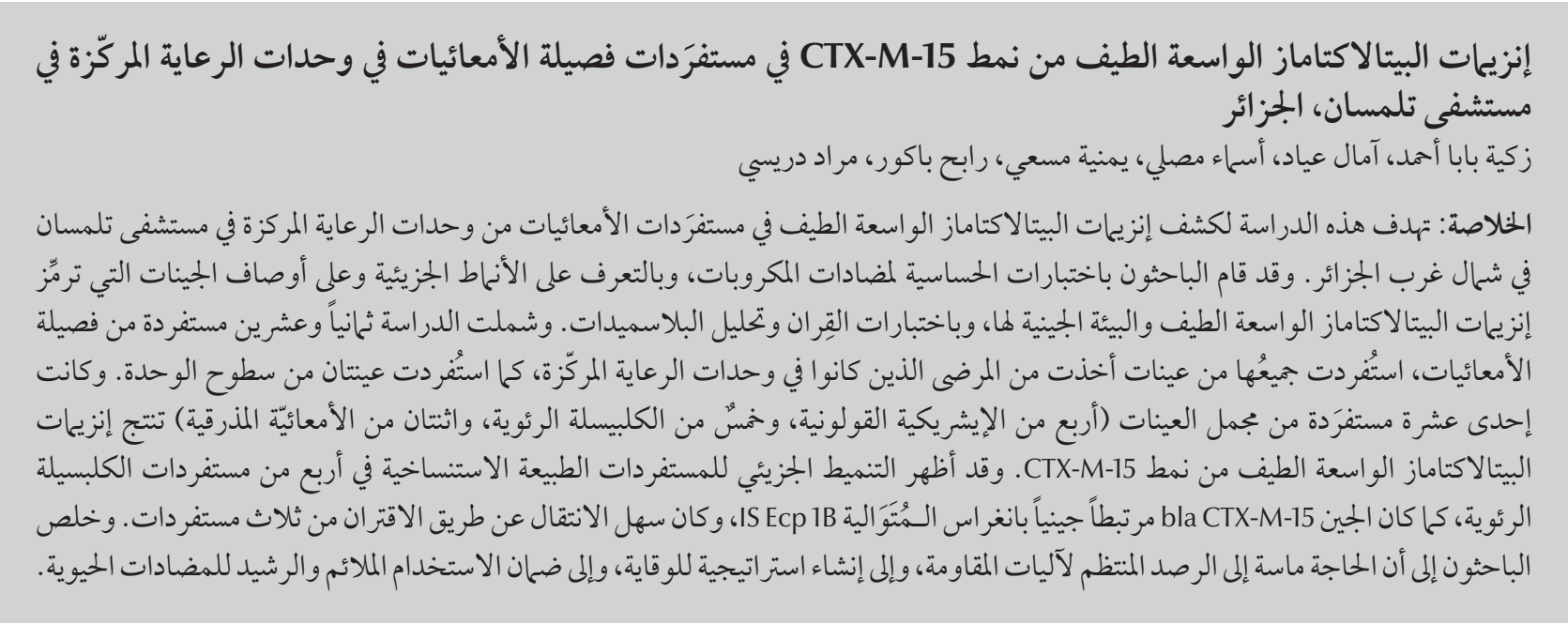

ABSTRACT The aim of this study was to detect extended-spectrum $\beta$-lactamases (ESBL) in Enterobacteriaceae isolates in the intensive care unit (ICU) of Tlemcen hospital in north-western Algeria. Antimicrobial susceptibility testing, molecular typing, characterization of ESBL-encoding genes and the genetic environment, conjugation experiments and plasmid analysis were carried out. In all, 28 Enterobacteriaceae isolates were isolated from specimens recovered from patients in the ICU and 2 from surfaces of the unit. Of these, 11 isolates (4 Escherichia coli, 5 Klebsiella pneumoniae and 2 Enterobacter cloacae) produced ESBL of the CT-X-M-15 type. Molecular typing of the isolates showed the clonal nature of $4 \mathrm{~K}$. pneumoniae isolates. The $b / a_{\mathrm{CTXM}-15}$ gene was genetically linked to insertion sequence ISECP1B and was transferable by conjugation from 3 isolates. Regular monitoring of resistance mechanisms, the establishment of a prevention strategy, and more rational and appropriate use of antibiotics are needed.

Détection de $\beta$-lactamases CTX-M-15 à spectre élargi chez Enterobacteriaceae dans l'unité de soins intensifs de I'hôpital de Tlemcen (Algérie)

RÉSUMÉ La présente étude visait à détecter des $\beta$-lactamases à spectre élargi dans des isolats d'Enterobacteriaceae au sein de l'unité de soins intensifs de l'hôpital de Tlemcen (nord-ouest de l'Algérie). Des tests de sensibilité aux antimicrobiens, un typage moléculaire, la caractérisation des gènes codants de $\beta$-lactamases à spectre élargi et de l'environnement génétique, des expériences de conjugaison et une analyse des plasmides ont été réalisés. Au total, 28 souches d'Enterobacteriaceae ont été isolées à partir d'échantillons prélevés chez des patients de l'unité de soins intensifs, et deux souches provenaient des surfaces de l'unité. Parmi celles-ci, onze isolats (quatre Escherichia coli, cinq Klebsiella pneumoniae et deux Enterobacter cloacae) ont produit des $\beta$-lactamases à spectre élargi de type CTX-M-15. Le typage moléculaire des souches a révélé la nature clonale de quatre isolats de $K$. pneumoniae. Le gène bla ${ }_{\mathrm{CTXM-15}}$ était génétiquement lié à la séquence d'insertion ISEcp1B et était transférable par conjugaison à partir de trois isolats. Une surveillance régulière des mécanismes de résistance, l'élaboration d'une stratégie préventive et l'usage plus rationnel et opportun des antibiotiques sont requis.

'Laboratory "Antibiotics-Antifungals", University of Abou Bekr Belkaïd, Tlemcen, Algeria.

${ }^{2}$ Laboratory of Cellular and Molecular Biology, University of Sciences and Technology Houari Boumédiène, Bab Ezzouar, Algiers, Algeria (Correspondence to R. Bakour: rbakour@yahoo.fr).

Received: 06/06/10; accepted: 02/03/11 


\section{Introduction}

$\beta$-lactams are the most common drugs against bacterial infections and the emergence of resistance to expanded-spectrum cephalosporins is a major concern $[1,2]$. The production of extended-spectrum $\beta$-lactamases (ESBLs) is a significant mechanism of resistance to $\beta$-lactams in Enterobacteriaceae; it has been found in the community, mostly in hospitals and often in intensive care units (ICUs) [3]. ESBLs are plasmid-mediated clavulanatesusceptible enzymes of predominantly Bush class A, capable of hydrolyzing oxyimino-cephalosporins and monobactams but not cephamycins and carbapenems. Typically, they derive from the genes of old $\beta$-lactamases TEM-1, TEM-2 or SHV-1 by mutation. However, new classes of ESBL have emerged such as PER, VEB, TLA-1, GES/IBC, SFO-1, BES-1, CTX-M [3].

CTX-M type $\beta$-lactamases have been widely detected around the world $[2,3]$. They were named thus because of their high level of activity against cefotaxime. The prevalence of the different types ESBLs varies by the clinical context and region and is changing over time $[2,3]$.

Algeria is a large country with over $1000 \mathrm{~km}$ of coastline and extending $2000 \mathrm{~km}$ to the south. All the studies that relate to ESBLs have only been conducted in the centre and east of the country. In this context, the objective of this qualitative study was to investigate the presence and the types of ESBLs in clinical isolates taken from the ICU of the university hospital of Tlemcen, a town located in the extreme north-west of the country, $600 \mathrm{~km}$ from the centre.

\section{Methods}

\section{Bacterial isolates, antimicrobial susceptibility testing and molecular typing}

From 19 October to 23 November 2008, 28 Enterobacteriaceae clinical isolates were isolated from various specimens recovered from patients in the ICU of Tlemcen university hospital; 2 cefotaxime-resistant isolates were also recovered from surfaces of the ICU. The patients had lung congestion, burning upon urination and fever.

Isolates were identified by using API 20E (BioMérieux, France). The antimicrobial susceptibility was determined by the disc diffusion and agar dilution methods according to CA-SFM (Comité de l'Antibiogramme de la Société Française de Microbiologie) guidelines [4]. Antibiotic disks were purchased from Bio-Rad (Marnes la Coquette, France). Escherichia coli ATCC 25922 was used as a control strain. ESBL production was screened by the doubledisc synergy test (DDST) [5]. The clonal relationships between isolates were analysed by enterobacterial repetitive intergenic consensus polymerase chain reaction (ERIC-PCR) using primer ERIC2 [6]. DNA was extracted by a boiling method as follows: $1.5 \mathrm{~mL}$ of an overnight broth culture were centrifuged at $15000 \times \mathrm{g}$ for $3 \mathrm{~min}$. The pellet was resuspended in $100 \mu \mathrm{L}$ of sterile ultrapure water and was then boiled for $10 \mathrm{~min}$. After a final centrifugation at $15000 \times \mathrm{g}$ for $10 \mathrm{~min}$, the supernatant was recovered. Thermal cycling was carried out as follows: an initial denaturation cycle at $95^{\circ} \mathrm{C}$ for $3 \mathrm{~min}, 40$ cycles of denaturation at $92^{\circ} \mathrm{C}$ for $30 \mathrm{~s}$, annealing at $40^{\circ} \mathrm{C}$ for $1 \mathrm{~min}$, extension cycle at $72^{\circ} \mathrm{C}$ for $8 \mathrm{~min}$, and a single final extension cycle at $72{ }^{\circ} \mathrm{C}$ for $16 \mathrm{~min}$. PCR products were separated by gel electrophoresis on $1.5 \%$ agarose in $1 \times$ Tris/borate/EDTA buffer, stained with ethidium bromide, visualized with a ultraviolet transilluminator. Fingerprints were visually compared and patterns differing by at least one amplification band were classified as different.

\section{Characterization of ESBL- encoding genes and genetic environment}

ESBL genes were identified by PCR as previously described using universal primers of the CTX-M family and specific primers for CTX-M groups (CTX-M-1, CTX-M-2, CTX-M-8, CTX-M-9, CTX-M-25) [7]. The PCR products were sequenced and the nucleotide sequences and deducedprotein sequences were analysed with the BLAST and FASTA programs of the National Center for Biotechnology Information (http://www.ncbi.nlm. nhi.gov).

Detection of the ISEcp $1 B$ sequence was performed by PCR using primers derived from the ISEcp $1 B$ transposase gene. The screening for a genetic linkage between ISEcp $1 B$ and ESBL genes was conducted by PCR using a combination of PROM+/CTXMB primers [8]. E. coli TN03, carrying an ISEcp 1-like element upstream from $b a_{\mathrm{CTX}-\mathrm{M}-15}$ gene, was used as a control.

\section{Conjugation experiments}

Mating experiments were performed as previously described [9] with E. coli C600 (rifampicin resistant) as a recipient. Selective agents were used at the following concentrations: $200 \mathrm{mg} / \mathrm{L}$ for rifampicin and $8 \mathrm{mg} / \mathrm{L}$ for cefotaxime. Transconjugants were subjected to antibiotic susceptibility testing, DDST and PCR analysis.

\section{Plasmid analysis}

Plasmid DNA was extracted by an alkaline lysis method as previously described $[10]$ and analysed by electrophoresis on $0.7 \%$ (wt $/ \mathrm{vol}$.) agarose gels at $5 \mathrm{~V} / \mathrm{cm}$. Plasmid size was estimated by using reference plasmids RP4 (60 kb) and pIP113 (128kb).

\section{Results}

The results are summarized in Table 1. Nine isolates (32.1\%) (E. coli, $n=$ 4; Klebsiella pneumoniae, $n=4$ and Enterobacter cloacae, $n=1$ ) of the 28 Enterobacteriaceae clinical isolates and the 2 selected isolates from surfaces ( $K$. pneumoniae and Ent. cloacae) showed a resistance to ESBL marker antibiotics (cefotaxime, ceftazidime, cefepime 


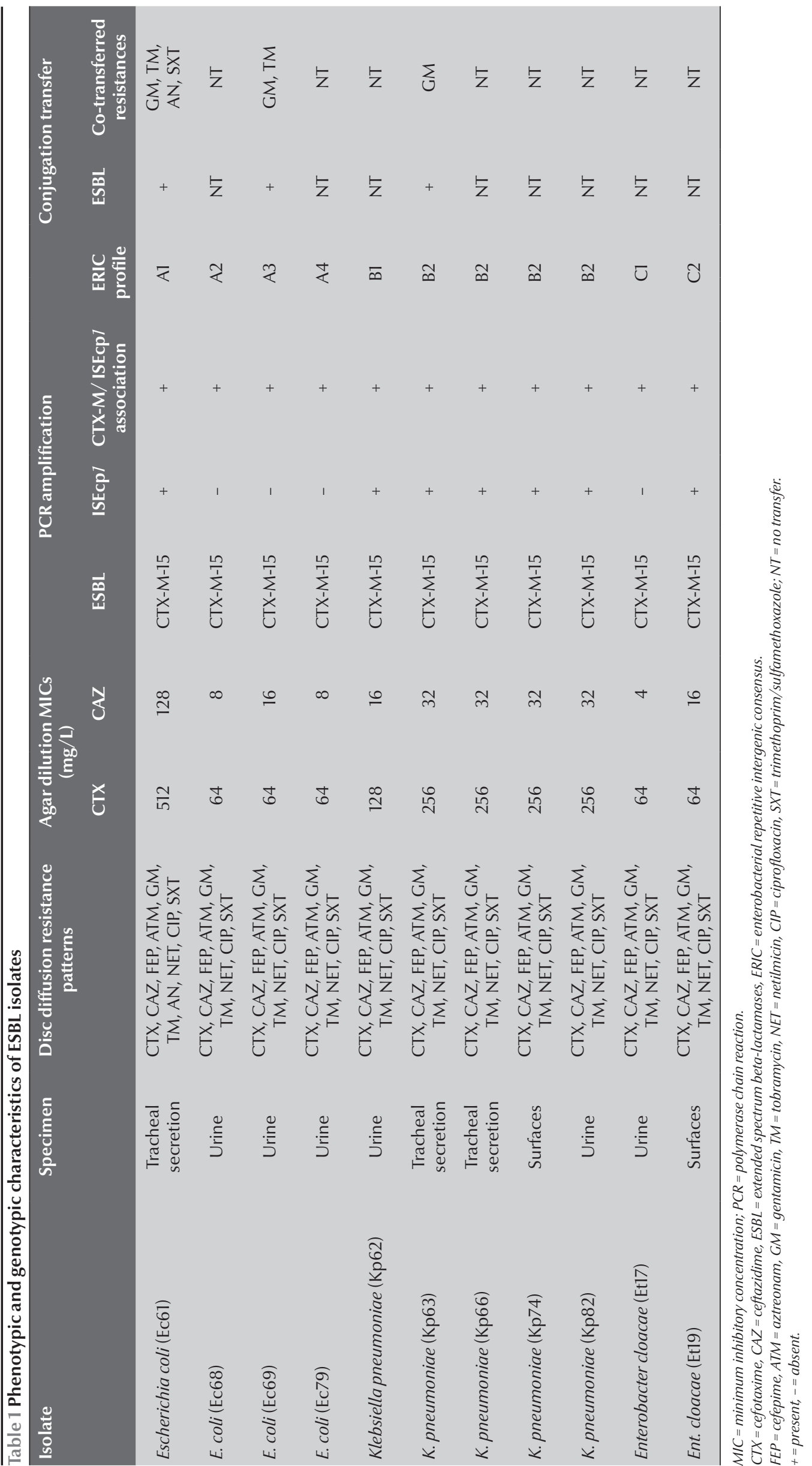


and aztreonam), a susceptibility to imipenem and a marked synergistic effect between clavulanic acid and the marker antibiotics, characteristic features of ESBL-producing bacteria. Agar dilution minimum inhibitory concentrations (MIC) of cefotaxime and ceftazidime were from 64 to 512 $\mathrm{mg} / \mathrm{L}$ and 4 to $128 \mathrm{mg} / \mathrm{L}$ respectively. These ESBL producers showed resistance to aminoglycosides (gentamicin, tobramycin and netilmicin), ciprofloxacin and trimethoprim-sulfamethoxazole. ERIC-PCR genotyping gave different electrophoresis patterns for E. coli and Ent. cloacae isolates, whereas $4 \mathrm{~K}$. pneumoniae isolates, one of which was from the surfaces, had identical profiles.

PCR amplification and sequencing revealed that all isolates produced ESBL of the CTX-M-15 type belonging to CTX-M-1 group. Insertion sequence ISEcp $1 B$ was found upstream of the bla $a_{\text {CTX-M-15 }}$ gene in 7 isolates including the $5 \mathrm{~K}$. pneumoniae, 1 E. coli and 1 Ent cloaceae; the genetic linkage investigation between this sequence and $b a_{\text {CTX-M-15 }}$ gene was positive for all isolates with PCR product of $1000 \mathrm{bp}$ being yielded.

Mating assays allowed the transfer of ESBL phenotype (oxyiminocephalosporin resistance) from 3 isolates including 2 E. coli and $1 \mathrm{~K}$. pneumoniae in association with $b a_{\text {CTX-M-15 }}$ gene and plasmids of about $90 \mathrm{~kb}$. Resistance determinants against the following nonlactam antibiotics were co-transferred: gentamicin; gentamicin-tobramycin and gentamicin-tobramycin-amikacintrimethoprim/sulfamethoxazole.

\section{Discussion}

In total, 11 Enterobacteriaceae isolates resistant to 3rd generation cephalosporins were ESBL producers; they were positive for the DDST and $b l a_{\text {CTX-M-15 }}$ gene. The presence of ESBL bacteria in the ICU of Tlemcen Hospital may be related to the fact that cefotaxime is the first-line treatment; in fact, the heavy use of cefotaxime and ceftriaxone is considered a factor supporting the emergence of CTX-M enzymes [11]. CTX-M-15 with CTX-M-3 enzymes have been reported as prevalent ESBLs in the east $[12]$ and centre $[6,13,14]$ of Algeria, while only CTX-M-15 was found in our isolates. The fact is that CTX-M enzymes have recently and sharply accumulated in Enterobacteriaceae [1]. The presence of CTX-M-15 enzyme in the west of Algeria indicates a countrywide spread of the CTX-M $\beta$-lactamases. This situation is comparable to those reported in numerous countries, such as Argentina, Poland and Lebanon where CTX-M-producing Enterobacteriaceae have been described to be endemic [15-18].

CTX-M-15 enzyme, like CTXM-16 and CTX-M-19, confers a higher resistance to ceftazidime than other types of CTX-M ESBLs. However, some of our isolates were moderately resistant to ceftazidime; this is in agreement with studies that described CTXM-15-producing isolates susceptible or moderately resistant to ceftazidime $[19,20]$. Resistance to aminoglycosides (gentamicin, tobramycin and netilmicin), ciprofloxacin and trimethoprim/ sulfamethoxazole was observed in all the isolates and transconjugant analysis showed the co-transfer of these resistances with CTX-M-15. The close association of ESBL production with these resistances has been previously reported $[7,19,21]$.

Genetic environment analysis of the CTX-M gene showed the presence of the sequence ISEcp $1 B$ in 7 isolates, while the genetic association between them was positive for all strains. The fact that ISEcp 1B was not detected in $3 E$. coli and E. cloacae isolates may be related to the modification in its transposase gene, possible by insertion of IS26 [22]. ISEcp $1 B$ can enhance the expression of the $b l a_{\text {CTX-M-15 }}$ gene and its presence could explain the ease with which this gene is spreading among bacteria in a clinical setting [8].

ERIC-PCR genotyping demonstrated the clonal diffusion of $K$. pneumoniae isolates. Epidemiological investigation has revealed that patients infected by these clones underwent invasive procedures (artificial ventilation and installation of probes). The detection of CTX-M ESBL in the remaining non-clonal E. coli and E. cloacae isolates is probably due to horizontal transmission via plasmids. Several nosocomial outbreaks caused by endemic or epidemic ESBL (CTX-M)-producing K. pneumoniae have been described, particularly in ICUs [23]. According to previous work, this is related to the misuse of broad spectrum antibiotics, invasive procedures and the immune status of patients in these care units [24].

\section{Conclusion}

This study demonstrated the presence of CTX-M-15 allele within Enterobacteriaceae in a hospital in north-western Algeria and suggests that its dissemination is associated with the spread of clonal isolates, plasmids and ISEcp1B. These data complement those of studies conducted in other areas of the country. There is a need for regular monitoring of resistance mechanisms and the establishment of a prevention strategy combining strict compliance to hygiene rules and a more rational and appropriate use of antibiotics.

\section{Acknowledgements}

This work was supported by a grant from "Fond National de la Recherche" of the Algerian Ministry of Higher Education and Scientific Research.

We thank Professor M.L. Joly-Guillou and Professor J. Cottin, Laboratoire de Bactériologie-Hygiène du Centre Hospitalier, Universitaire d'Angers, for their help in DNA sequencing. 


\section{References}

1. Livermore DM, Woodford N. The $\beta$-lactamase threat in Enterobacteriaceae, Pseudomonas and Acinetobacter. Trends in Microbiology, 2006, 14:413-414.

2. Hawkey PM, Jones AM. The changing epidemiology of resistance. Journal of Antimicrobial Chemotherapy, 2009, 64(Suppl. 1):3-10.

3. Bonnet R. Growing group of extended-spectrum beta-lactamases. The CTX-M enzymes. Antimicrobial Agents and Chemotherapy, 2004, 48:1-14.

4. CA-SFM. Comité de l'Antibiogramme de la Société Française de Microbiologie (2008) (http://www.sfm-microbiologie.org, accessed 5 March 2012).

5. Jarlier $\mathrm{V}$ et al. Extended broad- spectrum beta-lactamases conferring transferable resistance to newer $\beta$-lactam agents in Enterobacteriaceae: hospital prevalance and susceptibility patterns. Reviews of Infectious Diseases, 1988, 4:143-149.

6. Decré $\mathrm{D}$ et al. Outbreak of multiresistant Klebsiella oxytoca involving strains with extended-spectrum $\beta$-lactamase. Journal of Antimicrobial Chemotherapy, 2004, 54:881-888.

7. Messai $Y$ et al. Prevalence and characterisation of extended-spectrum beta-lactamases in Klebsiella pneumoniae in Algiers hospitals (Algeria). Pathologie Biologie, 2008, 56:319-325.

8. Poirel L, Decousser JW, Nordmann P. Insertion sequence ISEcp1B is involved in expression and mobilization of a bla $a_{\mathrm{CTX}-\mathrm{M}}$ $\beta$-lactamase gene. Antimicrobial Agents and Chemotherapy, 2003, 47:2938-2945.

9. Bakour R, Laroche Y, Cornelis G. Study of the incompatibility and replication of the 70-kb virulence plasmid of Yersinia. Plasmid, 1983, 10:279-289.

10. Kado Cl, Liu ST. Rapid procedure for detection and isolation of large and small plasmids. Journal of Bacteriology, 1981, 45:1365-1373.

11. Wang $\mathrm{H}$ et al. Clinical isolates of Enterobacteriaceae producing extended-spectrum $\beta$-lactamases: prevalence of CTX-M-3 at a hospital in China. Antimicrobial Agents and Chemotherapy, 2003, 47:790-793.

12. Touati $A$ et al. First report of CTX-M-15 and CTX-M-3 betalactamases among clinical isolates of Enterobacteriaceae in Béjaia, Algeria. International Journal of Antimicrobial Agents, 2006, 27:397-402.
13. labadene $\mathrm{H}$ et al. Dissemination of ESBL and Qnr determinants in Enterobacter cloacea in Algeria. Journal of Antimicrobial Chemotherapy, 2008, 62:133-136.

14. Ramdani-Bouguessa N et al. CTX-M-3 and CTX-M-15 extendedspectrum $\beta$-lactamases in isolates of Escherichia coli from a Hospital in Algiers, Algeria. Journal of Clinical Microbiology, 2006, 44:4584-4586.

15. Quinteros M. Extended-spectrum $\beta$-lactamases in Enterobacteriaceae in Buenos Aires, Argentina, Public Hospitals. Antimicrobial Agents and Chemotherapy, 2003, 47:2864-2867.

16. Baraniak A et al. Countrywide spread of CTX-M-3 extendedspectrum $\beta$-lactamase-producing microorganisms of the family Enterobacteriaceae in Poland. Antimicrobial Agents and Chemotherapy, 2002, 46:151-159.

17. Moubareck $\mathrm{C}$ et al. Countrywide spread of community- and hospital-acquired extended-spectrum $\beta$-lactamase (CTXM-15)-producing Enterobacteriaceae in Lebanon. Journal of Clinical Microbiology, 2005, 43:3309-3313.

18. Kanj SS et al. Molecular characterisation of extended-spectrum beta-lactamase-producing Escherichia coli and Klebsiella spp. isolates at a tertiary-care centre in Lebanon. Clinical Microbiology and Infection, 2008, 14:501-504.

19. Gangoue-Pieboji J et al. Emergence of CTX-M-15-producing Enterobacteriaceae in Cameroon and characterization of a bla ${ }_{\text {CTX-M-15 }}$-carrying element. Antimicrobial Agents and Chemotherapy, 2005, 49:441-443.

20. Woodford $\mathrm{N}$ et al. Community and hospital spread of Escherichia coli producing CTX-M extended-spectrum $\beta$-lactamases in the UK. Journal of Antimicrobial Chemotherapy, 2004, 54:735-743.

21. Lewis JS et al. First report of the emergence of CTX-M-type extended-spectrum-lactamases (ESBLs) as the predominant ESBL isolated in a U.S. health care system. Antimicrobial Agents and Chemotherapy, 2007, 51:4015-4021.

22. Eckert C, Gautier V, Arlet G. DNA sequence analysis of the genetic environment of various bla $a_{\mathrm{CTX}-\mathrm{M}}$ genes. Journal of Antimicrobial Chemotherapy, 2006, 57:14-23.

23. Silva $J$ et al. Outbreak of infection with extended-spectrum beta-lactamase-producing Klebsiella pneumoniae in a Mexican hospital. Journal of Clinical Microbiology, 2001, 39:3193-3196.

24. Kollef MH, Fraser $\mathrm{VJ}$. Antibiotic resistance in the intensive care unit. Annals of Internal Medicine, 2001, 134:298-314. 